\title{
ANÁLISE ESPAÇO-TEMPORAL DA COVID-19 EM UM ESTADO BRASILEIRO
}

\author{
SPACE-TIME ANALYSIS OF COVID-19 \\ IN A BRAZILIAN STATE
}

\section{ANÁLISIS ESPACIO-TIEMPO DE COVID-19 EN UN ESTADO BRASILEÑO}

\author{
Priscilla Dantas Almeida ${ }^{1}$ \\ Telma Maria Evangelista de Araújo ${ }^{2}$ \\ Augusto Cezar Antunes de Araújo Filho ${ }^{3}$ \\ Anderson Fuentes Ferreira ${ }^{4}$ \\ Inês Fronteira ${ }^{5}$ \\ Eugênio Barbosa de Melo Júnior ${ }^{6}$ \\ Malena Gonçalves Almeida ${ }^{7}$
}

Como citar este artigo: Almeida PD, Araújo TME, Araújo Filho ACA, Ferreira AF, Fronteira I, Melo Júnior EB, et al. Análise espaço-temporal da COVID-19 em um estado brasileiro. Rev baiana enferm. 2021;35:e42740.

Objetivo: analisar a distribuição espaço-temporal da COVID-19 no estado do Piauí. Método: estudo ecológico, retrospectivo, com dados disponíveis no Painel Epidemiológico COVID-19 - Piauí. Foi construída uma série temporal dos casos e óbitos acumulados mensalmente e calculadas as taxas de incidência, mortalidade e letalidade e construídos mapas cloropléticos utilizando-se o Quantum GIS, versão 2.18.6. Resultados: em março de 2020 foram registrados três casos, sem óbito, chegando a setembro de 2020 com 90.370 casos e 2.037 óbitos, com leve redução no crescimento das taxas a partir de agosto. Teresina apresentou o segundo menor coeficiente de incidência do estado, o segundo maior coeficiente de mortalidade e a maior letalidade. Conclusão: houve amplo crescimento da pandemia no estado, especialmente até o mês de agosto de 2020, com letalidade dentro do esperado, e a distribuição espacial dos casos e óbitos concentrada na capital e adjacências, evidenciando a necessidade de adoção de fortes medidas preventivas.

Descritores: Infecções por Coronavírus. Mortalidade. Incidência. Epidemiologia.

Objective: to analyze the spatial-temporal distribution of COVID-19 in the state of Piaui. Method: ecological, retrospective study, with data available from the COVID-19 Epidemiological Panel-Piaui. A time series of cases and deaths accumulated monthly was constructed and incidence, mortality and lethality rates were calculated and choropleth maps were constructed using Quantum GIS, version 2.18.6. Results: in March 2020, three cases were recorded, without death, reaching September 2020 with 90,370 cases and 2,037 deaths, with a slight reduction in the growth of rates from August. Teresina presented the second lowest incidence coefficient of the state, the second

\footnotetext{
Enfermeira. Mestre em Saúde e Comunidade. Universidade Federal do Piauí. Teresina, Piauí, Brasil. priscilladant@hotmail.com. http://orcid.org/0000-00026574-6335.

Enfermeira. Doutora em Enfermagem. Professora Associada da Universidade Federal do Piauí. Teresina, Piaú, Brasil. http://orcid.org/0000-000 I-5628-9577.

Enfermeiro. Doutor em Enfermagem. Professor Adjunto da Universidade Estadual do Piauí, Campus Doutora Josefina Demes. Floriano, Piauí, Brasil. http://orcid. org/0000-0002-3998-2334

Analista de sistemas. Mestre em Saúde Pública. Universidade Federal do Ceará. http://orcid.org/0000-0002-18|6-9459.

5 Enfermeira. Doutora em Saúde Internacional. Professora do Instituto de Higiene e Medicina Tropical da Universidade Nova de Lisboa. Lisboa, Portugal. http://orcid. org/0000-0003-1406-4585.

Enfermeiro. Mestre em Ciências e Saúde. Universidade Federal do Piauí. Teresina, Piaú, Brasil. http://orcid.org/0000-0002-0444-2960

Fisioterapeuta. Mestre em Saúde da Família. Fisioterapeuta na Secretaria de Estado da Saúde do Piauí. Teresina, Piauí, Brasil. http://orcid.org/0000-000 I-6583- I778.
} 
highest mortality coefficient and the highest lethality. Conclusion: there was a wide growth of the pandemic in the state, especially until August 2020, with lethality within the expected, and the spatial distribution of cases and deaths concentrated in the capital and surroundings, evidencing the need for strong preventive measures.

Descriptors: Coronavirus Infections. Mortality. Incidence. Epidemiology.

Objetivo: analizar la distribución espacial-temporal del COVID-19 en el estado de Piauí. Método: estudio ecológico y retrospectivo, con datos disponibles en el Panel Epidemiológico COVID-19 - Piauí. Se construyó una serie temporal de casos y muertes acumuladas mensualmente y se calcularon las tasas de incidencia, mortalidad y letalidad y se construyeron mapas coropléticos utilizando Quantum GIS, versión 2.18.6. Resultados: en marzo de 2020, se registraron tres casos, sin defunción, alcanzando septiembre de 2020 con 90.370 casos y 2.037 muertes, con una ligera reducción en el crecimiento de las tasas a partir de agosto. Teresina presentó el segundo coeficiente de incidencia más bajo del estado, el segundo coeficiente de mortalidad más alto y la mayor letalidad. Conclusión: hubo un amplio crecimiento de la pandemia en el estado, especialmente hasta agosto de 2020, con letalidad dentro de lo esperado, y la distribución espacial de casos y muertes concentrada en la capital y adyacencias, lo que evidencia la necesidad de fuertes medidas preventivas.

Descriptores: Infecciones por Coronavirus. Mortalidad. Incidencia. Epidemiología.

\section{Introdução}

O SARS-CoV-2 é um vírus que possui material de Ácido Ribonucleico (RNA) fita simples, responsável pelo Coronavírus Disease-2019 (COVID-19), considerada como o maior desafio da saúde pública mundial deste século. Trata-se de uma doença infecciosa capaz de provocar a Síndrome Respiratória Aguda Grave (SARS), com várias complicações e levar o indivíduo a óbito. A doença foi identificada pela primeira vez em Wuhan, na China, em dezembro de 2019. Em 19 de março de 2020, apesar dos inúmeros esforços da população chinesa, o país acumulava 81.174 casos confirmados da doença e 3.242 óbitos $^{(1-2)}$.

No Brasil, o primeiro caso da COVID-19 foi notificado em 26 de fevereiro de 2020, em homem, idoso, residente em São Paulo (SP) e vindo da Itália. A doença propagou-se rapidamente pelo país, e, em menos de um mês, foi considerada transmissão comunitária em todo o país ${ }^{(3)}$.

O estado do Piauí, localizado na Região Nordeste do Brasil, teve o primeiro caso confirmado da doença em 19 de março de 2020. Até o dia 31 de março do mesmo ano, o estado acumulava 18 casos e 4 óbitos provocados pelo novo coronavírus, resultando na letalidade de aproximadamente $22,2 \%$ e com a concentração dos casos na capital, Teresina ${ }^{(4)}$.
Frente ao exposto, tornou-se necessário analisar o atual cenário da doença no Estado após seis meses da notificação do primeiro caso. Informações quanto à incidência, mortalidade e letalidade, segundo as características epidemiológicas e espaço-temporais, tornam-se fundamentais para elaborar e direcionar as políticas e estratégias de enfrentamento da doença de forma mais positiva.

O presente estudo tem como objetivo analisar a distribuição espaço-temporal da COVID-19 no estado do Piauí.

\section{Método}

Trata-se de estudo ecológico, retrospectivo, com dados de março (primeiro registro da doença no Piauí) a setembro de 2020, mediante coleta de dados disponíveis no "Painel Epidemiológico COVID-19 - Piauŕ"(5). Optou-se por estudo ecológico, por este permitir analisar a ocorrência de determinada doença e a exposição entre populações para verificar a sua possível associação ${ }^{(6)}$.

Foram levantadas as variáveis referentes aos casos e óbitos por COVID-19 no estado do Piauí, acumulados por mês, para a construção da curva epidêmica e de óbitos, e também mês 
a mês para a construção de mapas cloropléticos, com vistas à melhor apresentação da evolução da doença.

Realizou-se a distribuição de casos e óbitos pelos onze territórios de desenvolvimento do estado do Piauí constantes no seu plano diretor de regionalização. Dentre os 224 municípios do estado do Piauí, foram identificados os 10 com maior número de casos e apresentadas as incidências cumulativas por 10.000 hab., a mortalidade por 100.000/hab. e a letalidade (\%) de cada um deles. Para caracterização, os óbitos foram distribuídos segundo o sexo, faixa etária e fatores de risco.

Para a distribuição espacial dos casos e óbitos, foram construídos mapas cloropléticos utilizando-se o Quantum GIS (QGIS), versão 2.18.6. Assim, a soma dos casos e óbitos foi organizada por mês e distribuídas por município de residência $(n=224$, divisão territorial de 2013). Para o cálculo das taxas, foi utilizada a população estimada para 2020, disponibilizada pelo Departamento de Informática do Sistema
Único de Saúde do Brasil (DATASUS). As taxas brutas foram calculadas dividindo-se os casos e óbitos pela população estimada, multiplicando por 100.000 habitantes. Para a categorização das classes espaciais das taxas brutas, utilizou-se o método de quebras naturais do algoritmo de classificação de Jenks (natural breaks).

A pesquisa foi submetida à apreciação do Comitê de Ética em Pesquisa (CEP), conforme Resolução nº 466/12, do Conselho Nacional de Saúde, e aprovada pelo Parecer no 4.088 .933 da Universidade Federal do Piauí.

\section{Resultados}

A primeira notificação da COVID-19 no Piauí foi registrada em 19 de março de 2020, com 3 casos da doença. Ao longo de seis meses, houve o aumento significativo dos casos, totalizando 90.370 casos em 19 de setembro de 2020. Em 19 de abril do referido ano já somavam 12 óbitos no estado, contabilizando, em setembro de 2020, 2.037 mortes por COVID-19 (Figura 1).

Figura 1 - Casos e óbitos acumulados após a primeira notificação de COVID no estado do Piauí. Teresina, Piauí, Brasil - 19 mar. 2020- 23 set. 2020

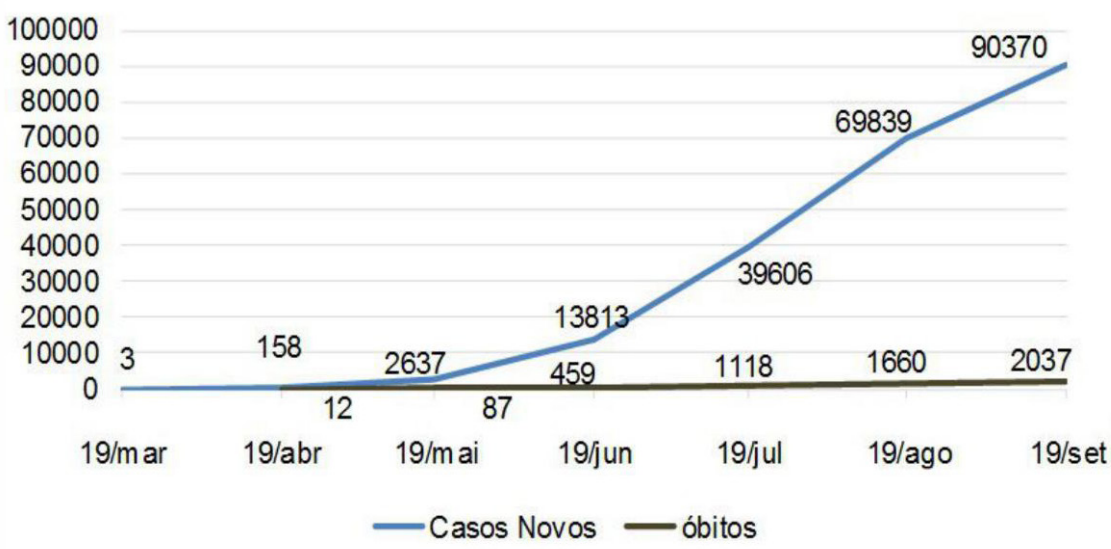

Fonte: Painel epidemiológico SESAPI ${ }^{(5)}$.

Foram notificados de 19 de março a 23 de setembro de 2020, 92.801 casos da COVID-19 no Piauí. Entre os 11 territórios de desenvolvimento do estado, verificou-se que as maiores frequências de casos e óbitos ocorreram no território de desenvolvimento Entre Rios (Tabela 1). 
Tabela 1 - Distribuição de casos e óbitos de COVID-19 por território de desenvolvimento do estado do Piauí. Teresina, Piauí, Brasil - 2020. ( N = 92.081)

\begin{tabular}{l|c|c|c|c}
\hline \multirow{2}{*}{ Territórios de desenvolvimento } & \multicolumn{2}{c|}{ Casos } & \multicolumn{2}{c}{ Óbitos } \\
\cline { 2 - 5 } & $\mathbf{n = 9 2 . 8 0 1}$ & $\mathbf{\%}$ & $\mathbf{n = 2 0 7 3}$ & $\mathbf{\%}$ \\
\hline Entre Rios & 41752 & 44,99 & 1196 & 57,69 \\
Cocais & 12666 & 13,65 & 231 & 11,14 \\
Planície Litorânea & 9560 & 10,30 & 203 & 9,79 \\
Vale do Rio Guaribas & 6876 & 7,41 & 113 & 5,45 \\
Tabuleiros do Alto Parnaíba & 4256 & 4,59 & 48 & 2,32 \\
Carnaubais & 3987 & 4,30 & 75 & 3,62 \\
Chapada das Mangabeiras & 3325 & 3,58 & 60 & 2,89 \\
Vale dos Rios Piauí e Itaueiras & 3300 & 3,56 & 55 & 2,65 \\
Vale do Canindé & 2900 & 3,12 & 37 & 1,78 \\
Serra da Capivara & 2500 & 2,69 & 24 & 1,16 \\
Vale do Sambito & 1679 & 1,81 & 31 & 1,50 \\
\hline
\end{tabular}

Fonte: Elaboração própria.

A Tabela 2 apresenta o ranking dos dez municípios com o maior número de casos. A capital, Teresina, apresentou o maior quantitativo de casos (30.795) e de óbitos (989) e maior taxa de letalidade (3,21\%). Entretanto, em relação ao coeficiente de incidência entre os dez municípios, Uruçuí apresentou o mais elevado (739,86/10.000 hab.). Quanto ao coeficiente de mortalidade, o de Barras foi o mais elevado (114,73/100.000 hab.).

Tabela 2 - Caracterização epidemiológica da COVID-19 nos municípios com maior quantitativo de casos no estado do Piauí. Teresina, Piauí, Brasil - 23 set. 2020. ( N = 92.081)

\begin{tabular}{l|c|c|c|c|c}
\hline \multirow{2}{*}{ Municipios } & \multicolumn{2}{|c|}{ Casos } & \multicolumn{3}{c}{ Óbitos } \\
\cline { 2 - 6 } & $\mathbf{n}$ & $\begin{array}{c}\text { Coeficiente de } \\
\text { Incidência* }\end{array}$ & $\mathbf{n}$ & $\begin{array}{c}\text { Coeficiente de } \\
\text { Mortalidade** }\end{array}$ & \% letalidade \\
\hline Teresina & 30795 & 356,08 & 989 & 114,36 & 3,21 \\
Parnaíba & 6673 & 435,92 & 135 & 88,19 & 2,02 \\
Picos & 3218 & 411,39 & 66 & 84,38 & 2,05 \\
Barras & 2551 & 542,00 & 54 & 114,73 & 2,12 \\
Floriano & 2493 & 415,95 & 35 & 58,40 & 1,40 \\
Campo Maior & 2295 & 490,04 & 43 & 91,82 & 1,87 \\
Piripiri & 2285 & 358,48 & 36 & 56,48 & 1,58 \\
Uruçuí & 1595 & 739,86 & 22 & 102,05 & 1,38 \\
Luzilândia & 1573 & 617,20 & 27 & 105,94 & 1,72 \\
Altos & 1436 & 354,36 & 23 & 56,76 & 1,60 \\
\hline
\end{tabular}

Fonte: Painel epidemiológico SESAPI ${ }^{(5)}$.

Notas:

*Incidência por $10.000 \mathrm{Hab}$.

**Mortalidade por 100.000 Hab.

No tocante às características epidemiológicas dos óbitos por COVID-19 no estado do Piauí, destacaram-se as prevalências: sexo feminino (57,6\%), faixa etária de 80 anos ou mais (32,66\%), cardiopatias, incluindo hipertensão como fator de risco (43,63\%), seguida de diabetes $(25,4 \%)$ (Tabela 3). 
Tabela 3 - Distribuição dos óbitos de COVID-19 segundo sexo, faixa etária e comorbidades no estado do Piauí. Teresina, Piauí, Brasil - 23 set. 2020. ( $\mathrm{N}=2.073)$

\begin{tabular}{|c|c|c|}
\hline Variáveis & $\mathbf{n}$ & $\%$ \\
\hline \multicolumn{3}{|l|}{ Sexo } \\
\hline Feminino & 1195 & 57,6 \\
\hline Masculino & 878 & 42,4 \\
\hline \multicolumn{3}{|l|}{ Faixa etária (anos) } \\
\hline 0 a 9 & 4 & 0,19 \\
\hline 10 a 19 & 8 & 0,39 \\
\hline 20 a 29 & 25 & 1,21 \\
\hline 30 a 39 & 62 & 2,99 \\
\hline 40 a 49 & 137 & 6,61 \\
\hline 50 a 59 & 224 & 10,81 \\
\hline 60 a 69 & 392 & 18,91 \\
\hline 70 a 79 & 544 & 26,24 \\
\hline 80 e mais & 677 & 32,66 \\
\hline \multicolumn{3}{|l|}{ Fatores de risco/comorbidade entre os óbitos* } \\
\hline Cardiopatias incluindo hipertensão & 904 & 43,63 \\
\hline Diabetes & 526 & 25,4 \\
\hline Sem comorbidade & 129 & 6,21 \\
\hline Doença neurológica crônica ou neuromuscular & 117 & 5,63 \\
\hline Pneumopatia & 98 & 4,73 \\
\hline Doença Renal & 98 & 4,73 \\
\hline Obesidade & 75 & 3,61 \\
\hline Neoplasia & 62 & 2,98 \\
\hline Imunodepressão & 24 & 1,15 \\
\hline Doença hepática & 20 & 0,99 \\
\hline Asma & 14 & 0,67 \\
\hline Síndrome de Down & 3 & 0,13 \\
\hline Doença hematológica & 2 & 0,11 \\
\hline Puérpera & 1 & 0,03 \\
\hline
\end{tabular}

Fonte: Painel epidemiológico SESAPI ${ }^{(5)}$.

Nota:

*Devido ao acesso apenas à frequência, foi realizada aproximação para definir o número absoluto.

De março ao último dia de setembro de 2020, foi possível contabilizar 2.127 óbitos. A taxa de óbitos apresentou um espalhamento da epidemia nos municípios. A expansão começou na capital, seguindo em direção aos municípios do interior. A partir de junho de 2020, o estado apresentou taxa de mortalidade superior a 27 óbitos por 100.000 habitantes. 
Figura 2 - Mapas com a distribuição da taxa de óbito por município segundo o mês de ocorrência. Teresina, Piauí, Brasil - 2020
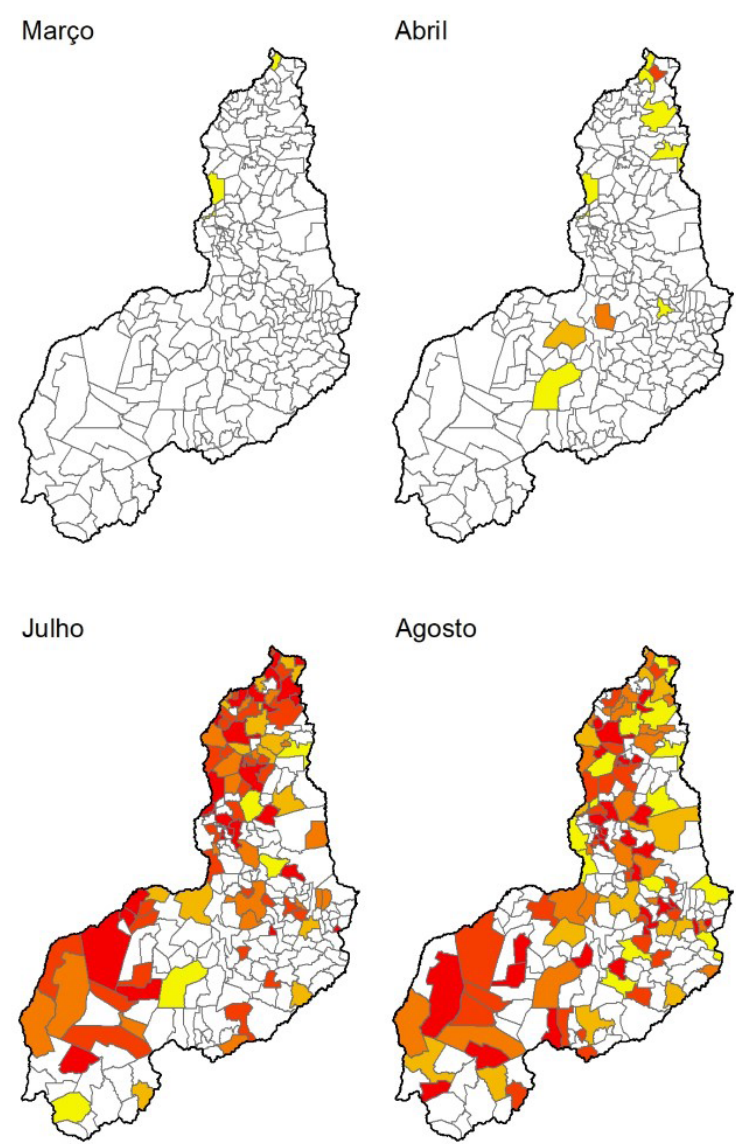
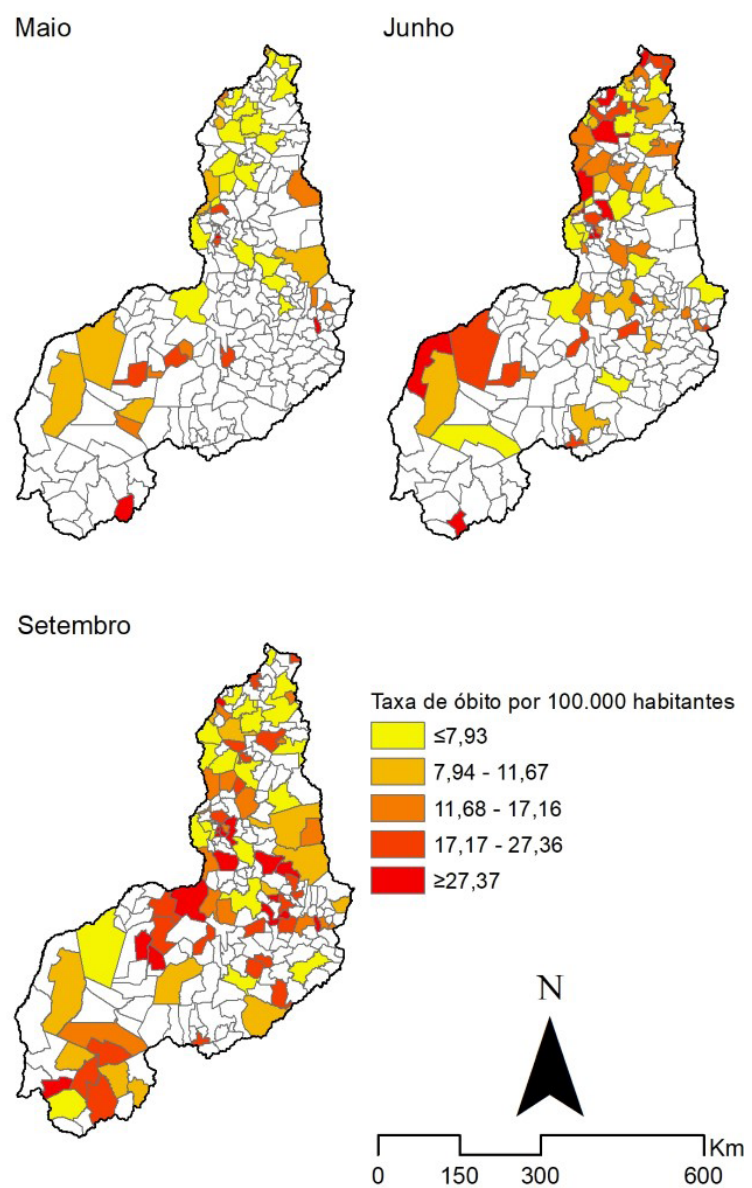

Fonte: Painel epidemiológico SESAPI ${ }^{(5)}$.

\section{Discussão}

A cidade de Teresina acumulou, no período analisado, o maior número absoluto de casos e óbitos por COVID-19, bem como apresentou a maior letalidade entre os municípios investigados e superior à do Brasil (3,0\%), conforme dados do Painel Coronavírus do Ministério da Saúde $^{(7)}$. Estudo chinês, com 44.672 casos confirmados, apresentou letalidade de 2,3\%, isto é, inferior à registrada em Teresina ${ }^{(8)}$.

Estudo que comparou a letalidade dos casos relatados da infecção pelo novo coronavírus, da síndrome respiratória aguda grave (SARS) e da síndrome respiratória do Oriente Médio (MERS-CoV) verificou que a taxa de letalidade da COVID-19 é próxima de 3\%, e não parece ser tão letal como as outras duas. Entretanto, o número total de

casos e de óbitos por COVID-19 já é bem superior que a SARS e a MERS-COV ${ }^{(9)}$.

Estudo realizado no Piauí identificou que o território Entre Rios possui 65\% (149) dos leitos de Unidade de Terapia Intensiva (UTI), e conta com a capital, Teresina, como cidade principal para o atendimento de alta complexidade do território. Esta pesquisa apontou que o território citado, mesmo possuindo o maior quantitativo de UTI, apresentou o maior número de óbitos e de casos da COVID-19. Isso pode ser explicado por Teresina concentrar a maior parte dos casos, devido às suas características de capital, como quantitativo populacional, acesso por rodovias e contar com aeroporto ${ }^{(4)}$.

A letalidade analisada, neste estudo, por cidade, e o coeficiente de mortalidade, por território, apresentam diferenças relevantes a 
depender do espaço, como evidenciado em estudo ecológico sobre as variações da letalidade por COVID-19 nos estados brasileiros, envolvendo todos os óbitos por COVID-19 por Unidade Federativa e na Semana Epidemiológica. Sugere-se, então, que esse achado pode estar relacionado às desigualdades sociais, estruturais e econômicas e iniquidades em saúde, presentes na sociedade, necessitando de políticas direcionadas às características de cada local ${ }^{(10)}$.

Ressalta-se, ainda, que as informações quanto ao número de óbitos podem sofrer influência das subnotificações, que ocorrem por inúmeros fatores, como realização de testagem correta conforme o tempo de apresentação de sintomas e o exame adequado para cada pessoa, além de diversos outros fatores intrínsecos dessa doença nova.

Ainda em relação aos óbitos por COVID-19, o sexo feminino foi predominante, fato que diverge de estudo internacional ${ }^{(11)}$, realizado em 169 hospitais de 11 países, nos quais os achados sugerem que as mulheres possuem maior probabilidade proporcional de sobreviver à infecção, devido à imunidade inata e adaptativa, relacionada ao cromossomo X e aos hormônios sexuais, o que torna as mulheres mais fortes e com maior resistência a infecções virais em relação aos homens ${ }^{(11-12)}$

A idade avançada (maior que 60 anos) apresenta-se como fator de risco para a mortalidade por COVID-19 ${ }^{(12)}$, o que corrobora os achados deste estudo. Por outro lado, as crianças de 0 a 9 anos contribuíram com a menor frequência dos casos, o que coaduna com a realidade de muitos outros estados e países. As crianças apresentam menor suscetibilidade à infecção pela COVID-19, em decorrência de múltiplos fatores, como o reduzido ciclo de amizades, não ter atividades trabalhistas, não estarem frequentando a escola, não saírem às compras, determinando uma convivência social restrita. O fato de as crianças serem consideradas potenciais carreadoras da infecção as colocou em situação de forte distanciamento social, limitando inclusive o contato com familiares pertencentes aos grupos de risco ${ }^{(13)}$.

Neste estudo, as comorbidades mais predominantes entre os óbitos por COVID-19 foram as cardiopatias (incluindo a hipertensão), seguida pelo diabetes. Esses achados corroboram estudo realizado nos Estados Unidos ${ }^{(14)}$ e comum à meta-análise ${ }^{(15)}$, nos quais a hipertensão e o diabetes foram as comorbidades mais prevalentes entre as mortes por COVID-19.

Condições pré-existentes como doenças cardiovasculares, doença renal crônica, doenças pulmonares crônicas (sobretudo a Doença Pulmonar Obstrutiva Crônica (DPOC)), Diabetes Mellitus, hipertensão, imunossupressão, obesidade e doença falciforme são apontadas como fatores que predispõem os indivíduos a um prognóstico clínico desfavorável, bem como aumentam o risco de intubação e morte ${ }^{(16)}$.

Estudo realizado com 1.590 pacientes na China apontou maior gravidade da COVID-19 em pacientes que possuem fatores de risco em comparação àqueles que não as possuem. Ademais, verificou que pacientes com duas ou mais comorbidades apresentaram risco de prognóstico ruim, expressivamente maior do que aqueles que não tinham ou tinham apenas uma comorbidade $^{(17)}$. Torna-se relevante considerar que essas condições e suas características de suscetibilidade podem estar relacionadas à patogênese da COVID-19 ${ }^{(18)}$.

Verificou-se que houve uma variação na taxa de mortalidade em cada mês nos municípios do Piauí, pois, cidade que no início da pandemia apresentava altas taxas de mortalidade, teve essa taxa reduzida em meses posteriores, assim como o contrário também foi identificado. Em março de 2020 foram registrados óbitos apenas em Teresina e Parnaíba, porém, com a interiorização da doença, houve o aumento dos óbitos em outras cidades. Achado semelhante foi observado em estudo realizado no Ceará, com a chegada da COVID-19 aos municípios mais distantes das metrópoles, tornando-se necessária a distribuição de leitos de cuidados intensivos destinados aos pacientes com a doença ${ }^{(19)}$. 
Infere-se que as taxas de óbitos elevadas em determinadas cidades revelam a situação deficitária do sistema de saúde para assistir a demanda crescente de pacientes com COVID-19 em alguns municípios. Logo, tornam-se necessárias medidas para a redução da propagação da doença, expansão dos leitos disponíveis e organização dos serviços de saúde de forma regionalizada no estado, a fim de reduzir a distância percorrida pelo paciente para chegar ao serviço de saúde ${ }^{(20)}$.

A principal limitação deste estudo está na abordagem pelo uso de dados secundários disponíveis pelas Secretarias de Saúde, que podem apresentar viés de informação, e por não permitir definir o comportamento da doença nos próximos meses. Estudos que trabalhem com análise espacial dos pacientes recuperados poderão contribuir para analisar a evolução da doença no estado do Piauí.

\section{Conclusão}

O estudo permitiu analisar a situação da doença por territórios de desenvolvimento e a distribuição aleatória dos óbitos nos municípios do estado. Observou-se uma rápida e ampla ascensão da pandemia, especialmente até o mês de agosto de 2020, com letalidade dentro do esperado. O padrão de distribuição espacial dos casos e, especialmente, dos óbitos apresentou concentração na capital e adjacências, evidenciando a necessidade de fortes medidas preventivas neste território de desenvolvimento.

Identificou-se prevalência da mortalidade na população idosa, pessoas do sexo feminino e pacientes com cardiopatia, incluindo a hipertensão. Relacionar as características epidemiológicas com os óbitos provocados pela COVID-19 no estado é fundamental para avaliar as medidas de controle da doença, assim como os cuidados aplicados até o momento. Possibilita, ainda, a definição de novas estratégias, protocolos e condutas frente a esses pacientes, na perspectiva de redução dos sintomas da doença, complicações e óbitos.
Recomenda-se a elaboração de planos que considerem as características sociais, culturais e econômicas dos diferentes territórios de desenvolvimento, com o objetivo de reduzir o impacto da pandemia e ampliar a efetividade das ações de controle.

\section{Financiamento:}

Esta pesquisa foi financiada pela empresa JBS por meio do "Projeto Fazer o Bem Faz Bem: impacto social durante a pandemia".

\section{Colaborações:}

1 - concepção, projeto, análise e interpretação dos dados: Priscilla Dantas Almeida, Telma Maria Evangelista de Araújo, Augusto Cezar Antunes de Araújo Filho, Anderson Fuentes Ferreira, Eugênio Barbosa de Melo Júnior e Malena Gonçalves Almeida;

2 - redação do artigo e revisão crítica relevante do conteúdo intelectual: Priscilla Dantas Almeida, Telma Maria Evangelista de Araújo, Augusto Cezar Antunes de Araújo Filho, Eugênio Barbosa de Melo Júnior e Malena Gonçalves Almeida;

3 - aprovação final da versão a ser publicada: Priscilla Dantas Almeida, Telma Maria Evangelista de Araújo, Augusto Cezar Antunes de Araújo Filho e Inês Fronteira.

\section{Referências}

1. Heymann DL, Shindo N. COVID-19: what is next for public health? Lancet. 2020; 395(10224):542-5. DOI: https://doi.org/10.1016/ S0140-6736(20)30374-3

2. Guangbiao Z, Saijuan C, Zhu C. Advances in COVID-19: the virus, the pathogenesis, and evidence-based control and therapeutic strategies. Front Med. 2020;14(2):117-25. DOI: https://doi. org/10.1007/s11684-020-0773-x

3. Oliveira WK, Duarte E, França GVA, Garcia LP. How Brazil can hold back COVID-19. Epidemiol Serv Saúde. 2020;29(2):e2020044. DOI: https://doi. org/10.5123/s1679-49742020000200023 
4. Batista FMA, Mascarenhas MDM, Marinelli NP, Albuquerque LPA, Rodrigues MTP, Vieira MACS, et al. COVID-19 in Piauí: initial scenario and perspectives for coping. Rev Soc Bras Med Trop. 2020;53:e20200175. DOI: https://doi.org/ $10.1590 / 0037-8682-0175-2020$

5. Piauí. Secretaria de Estado de Saúde. Painel Epidemiológico Covid-19 Piauí [Internet]. Teresina (PI); 2020 [cited 2020 Oct 6]. Available from: https://datastudio.google.com/reporting/ a6dc07e9-4161-4b5a-9f2a-6f9be486e8f9/ page/2itOB

6. Lima-Costa MF, Barreto SM. Tipos de estudos epidemiológicos: conceitos básicos e aplicações na área do envelhecimento. Epidemiol Serv Saúde. 2003;12(4):189-201. DOI: http://dx.doi. org/10.5123/S1679-49742003000400003

7. Brasil. Ministério da Saúde. COVID19 Painel Coronavírus [Internet]. Brasília (DF); 2020 [cited 2020 Oct 7]. Available from: https://covid.saude. gov.br/

8. Wu Z, McGoogan JM. Characteristics of and Important Lessons from the Coronavirus Disease 2019 (COVID-19) Outbreak in China: Summary of a Report of 72314 Cases from the Chinese Center for Disease Control and Prevention. JAMA. 2020;323(13):1239-42. DOI: https://doi. org/10.1001/jama.2020.2648

9. Pimentel RMM, Daboin BEG, Oliveira AG, Macedo Jr H. The dissemination of COVID-19: an expectant and preventive role in global health. J Hum Growth Dev. 2020;30(1):135-40. DOI: http://doi.org/10.7322/jhgd.v30.9976

10. Souza CDF, Paiva JPS, Leal TC, Silva LF, Santos LG. Evolução espaço temporal da letalidade por COVID-19 no Brasil, 2020. J Bras Pneumol. 2020;46(4):e20200208. DOI: https://doi. org/10.36416/1806-3756/e20200208

11. Mehra MR, Desai SS, Kuy S, Henry TD, Patel AN. Cardiovascular Disease, Drug Therapy, and Mortality in Covid-19. N Engl J Med. 2020;382:e102. DOI: https://doi.org/10.1056/ NEJMoa2007621

12. Long-quan L, Tian $\mathrm{H}$, Yong-qing W, Zheng-ping W, Yuan L, Tao-bi $\mathrm{H}$, et al. COVID-19 patients' clinical characteristics, discharge rate, and fatality rate of meta-analysis. J Med Virol. 2020;92:577-83. DOI: https://doi. org/10.1002/jmv.25757

13. Epidemiology Working Group for NCIP Epidemic Response, Chinese Center for Disease Control and Prevention. The epidemiological characteristics of an outbreak of 2019 novel coronavirus diseases (COVID-19) in China. Zhonghua Liu Xing Bing Xue Za Zhi. 2020;41(2):145-51. DOI: 10.3760/cma.j.issn.02546450.2020 .02 .003

14. Sanyaolu A, Okorie C, Marinkovic A, Patidar R, Younis K, Desai P, et al. Comorbidity and its Impact on Patients with COVID-19. SN Compr Clin Med. 2020;2(8):1069-76. DOI: https://doi. org/10.1007/s42399-020-00363-4

15. Espinosa OA, Zanetti AS, Antunes EF, Longhi FG, Matos TA, Battaglini PF. Prevalence of comorbidities in patients and mortality cases affected by SARS-CoV2: a systematic review and meta-analysis. Rev Inst Med trop S Paulo. 2020;62:e43. DOI: https://doi.org/10.1590/s16789946202062043

16. Marin BG, Aghagoli G, Lavine K, Yang L, Siff EJ, Chiang SS, et al. Predictors of COVID-19 severity: A literature review. Rev Med Virol. 2020;31(1):e2146. DOI: https://doi.org/10.1002/ rmv. 2146

17. Wei-jie G, Wen-hua L, Yi Z, Heng-rui L, Zi-sheng C, Yi-min L, et al. Comorbidity and its impact on 1590 patients with COVID-19 in China: a nationwide analysis. Eur Respir J. 2020;55(5):2000547. DOI: https://doi.org/10.1183/ $13993003.00547-2020$

18. Jing Y, Ya Z, Xi G, Ke P, Zhaofeng C, Qinghong $G$, et al. Prevalence of comorbidities and its effects in patients infected with SARS-CoV-2: a systematic review and meta-analysis. Int J Infect Dis. 2020;94:91-5. DOI: https://doi.org/10.1016/j.ijid.2020.03.017

19. Pedrosa NL, Albuquerque NLS. Spatial Analysis of COVID-19 cases and intensive care beds in the State of Ceará, Brazil. Ciênc saúde coletiva. 2020;25(Suppl 1):2461-8. DOI: https://doi.org/ 10.1590/1413-81232020256.1.10952020 
20. Noronha KVMS, Guedes GR, Turra CM, e00115320. DOI: https://doi.org/10.1590/0102-311 Andrade MV, Botega L, Nogueira D, et al. The COVID-19 pandemic in Brazil: analysis of supply and demand of hospital and ICU beds and mechanical ventilators under different scenarios. Cad Saúde Pública. 2020;36(6): x00115320

Recebido: 11 de dezembro de 2020 Aprovado: 18 de maio de 2021 Publicado: 8 de junho de 2021

A Revista Baiana de Enfermagem utiliza a Licença Creative Commons - Atribuição-NãoComercial 4.0 Internacional. https://creativecommons.org/licenses/by-nc/4.0/ Este artigo é de acesso aberto distribuído sob os termos da Licença Creative Commons (CC BY-NC). Esta licença permite que outros remixem, adaptem e criem a partir do seu trabalho para fins não comerciais. Embora os novos trabalhos tenham de lhe atribuir o devido crédito e não possam ser usados para fins comerciais, os usuários não têm de licenciar esses trabalhos derivados sob os mesmos termos. 